\title{
ASCA OBSERVATIONS OF NARROW LINE SEYFERT 1 GALAXIES
}

\author{
K. HAYASHIDA \\ Department of Earth 8 Space Science, Osaka University \\ 1-1, Machikaneyama, Toyonaka, Osaka,Japan \\ hayasida@ess.sci.osaka-u.ac.jp
}

\begin{abstract}
Study of nine Narrow Line Seyfert 1 galaxies (NLS1) observed with ASCA is presented. The X-ray spectra are fitted with a two component model. The soft component can be represented by a blackbody model with $\mathrm{kT} \sim 100 \mathrm{eV}$. To make the observed luminosity below the Eddignton limit, we have to assume the emission region has a size much smaller than 3Rs. The X-ray variabilities of the NLS1s have systematically shorter time scale than the typical (broad line) Seyferts 1 (BLS1), which suggests lower black hole masses in the NLS1s.

We also study the the X-ray spectral structure around $1.1 \mathrm{keV}$, which has been reported for some NLS1s. Although the structure has been interpreted as an absorption feature, we propose an alternative model employing emission lines. One possible origin of the emission lines is preprocessing from a highly ionized accretion disk.
\end{abstract}

\section{X-ray Spectra: Soft Component}

The NLS1s we have analyzed are IRAS13224, H0707, PG1404, Mkn478, RE1034, PG1211, Mrk766, IZw1, and PG1244 (Note that results of some sources have already been published by authors). The ASCA spectra (0.4$10 \mathrm{keV}$ ) of these NLS1s were fitted with a two component model (a soft component and a hard power law component), though inclusion of the soft component is not necessary for some sources (e.g. IZw1). We employed a blackbody model to fit the soft component. One important consequence is that the best fit blackbody temperatures concentrate around $\mathrm{kT} \sim 100 \mathrm{eV}$.

With the blackbody model fit, the size of the emission region was derived for each sources. If we assume it corresponds to $3 \mathrm{Rs}$, we can estimate the 
black hole mass, ranging from $2 \times 10^{4}$ to $10^{6} M_{o}$. Employing those mass values, however, leads to the super-Eddignton luminosity. Using a smaller radius, e.g. $0.5 \mathrm{Rs}$, the corresponding Eddington limit becomes larger by a factor of 6 and the observed soft component luminosity go in the Eddignton limit. This result may imply the Kerr metric for these sources if the soft component come from an optically thick accretion disk.

\section{X-ray Variability}

Rapid and large amplitude X-ray variability is one of the characteristics of the NLS1s. We employed the analysis method previously applied to BLS1s (Hayashida et al., 1997a). From the variability time scale, we estimated the central black hole masses of nine sources, ranging from $2 \times 10^{4}$ to $10^{7} M_{o}$. These mass values of the NLS1s are systematically lower than those for the BLS1s. We speculate that the lower black hole masses in the NLS1 is is one of the main causes for their peculiar properties among the Seyferts.

\section{X-ray Spectral Feature around $1.1 \mathrm{keV}$ : Emission Line Model}

In some NLS1s, a peculiar spectral structure was reported around $1.1 \mathrm{keV}$ so far(IRAS13224: Otani et al., 1996, PG1404:Comastri et al., 1997, H0707: Hayashida, 1997b). Although the feature has been considered as absorption feature (e.g. highly blue-shifted O edge, see Leighly et al., 1997), we propose an alternative interpretation, i.e. emission lines from ionized materials. For example, Gaussian emission lines with energies of $0.84( \pm 0.01), 0.99( \pm 0.01)$, and 1.66( \pm 0.03$) \mathrm{keV}$ improve the fit for IRAS13224. In the case of H0707, the energies are $0.68( \pm 0.02), 0.87( \pm 0.02)$, and $1.41( \pm 0.02) \mathrm{keV}$. One possible cause of the emission lines is fluorescent reprocessed from highly ionized matter on the surface of an accretion disks, such as indicated by Ross and Fabian (1993). Although detailed study is needed for the model fit, if this model is appropriate it means that we have a new probe to observe the status and the abundance of the matter very close to the central massive black hole.

\section{References}

Hayashida, K. et al. (1997a), $A p J$, accepted.

Otani, C., Kii, T. \& Miya, K. (1996) MPE report, p.263.

Comastori, A., Molendi, S. \& Ulrich, M.H. (1997) X-ray Imaging and Spectroscopyof Cosmic Hot Plasmas, p.279.

Hayashida, K. (1997b), Emission Line in Active Galaxies (IAU colloquium 159), p.40.

Leghily, K.M. et al. (1997) $A p J$, in press.

Ross, R.R. \& Fabian, A.C. (1993), MNRAS, 261, p.74. 